\title{
On Comparative Analogy between Ant Colony Systems and Neural Networks Considering Behavioral Learning Performance
}

\author{
Hassan M. H. Mustafa ${ }^{1, *}$, Ayoub Al-Hamadi ${ }^{2}$, Mohamed Abdulrahman ${ }^{3}$, Shahinaz Mahmoud ${ }^{4}$, \\ Mohammed O Sarhan \\ ${ }^{1}$ Computer Engineering Department, Faculty of Engineering, Al-Baha University, Al-Baha,KSA.On leave from Banha University, Egypt \\ ${ }^{2}$ Institute for Information Technology and Communications, Otto-von-Guericke-University Magdeburg, Germany \\ ${ }^{3}$ Electrical Engineering Department, Faculty of Engineering, Al-Baha University, Al-Baha, KSA \\ ${ }^{4}$ Instructional \& Information Technology Educational Technology Department, Women College,Ain Shams University, Cairo, Egypt \\ ${ }^{5}$ Al-Salt College of Human Sciences, Al- Balqa Applied University Hashemite Kingdom of Jordan \\ *Corresponding author: Prof.dr.hassanmoustafa@gmail.com
}

Received April 24, 2015; Revised May 22, 2015; Accepted June 26, 2015

\begin{abstract}
This article addresses an interesting comparative analytical study. The presented study considers two concepts of diverse algorithmic biological behavioral learning approach. Those concepts for computational intelligence are tightly related to neural and non-neural Systems. Respectively, the first algorithmic intelligent approach concerned with observed obtained practical results after three neural animal systems' activities. Namely, they are Pavlov's, and Thorndike's experimental work. Furthermore, a mouse's trials during its movement inside figure of eight (8) maze, those aiming to reach optimal solution for reconstruction problem. However, second algorithmic intelligent approach conversely originated from observed activities' results for non-neural Ant Colony System (ACS). Those results have been obtained after reaching optimal solution solving Traveling Sales-man Problem (TSP). Interestingly, the effect of increasing number of agents (either neurons or ants) on learning performance shown to be similar for both introduced neural and non-neural systems. Considering observed two systems' performances, it has shown both to be in agreement with learning convergence process searching for Least Mean Square (LMS) error algorithm. Accordingly, adopted ANN modeling is realistically relevant tool systematic observations' investigation and performance analysis for both selected computational intelligence (biological behavioral learning) systems.
\end{abstract}

Keywords: artificial neural network, behavioral learning, ant colony system, traveling salesman problem, and computational biology

Cite This Article: Hassan M.H. Mustafa, Ayoub Al-Hamadi, Mohamed Abdulrahman, Shahinaz Mahmoud, and Mohammed O Sarhan, "On Comparative Analogy between Ant Colony Systems and Neural Networks Considering Behavioral Learning Performance.” Journal of Computer Sciences and Applications, vol. 3, no. 3 (2015): 79-89. doi: 10.12691/jcsa-3-3-4.

\section{Introduction}

An investigational approach is adopted for comparative study of analogy between two simulated naturally inspired systems. Herein, this work introduces comprehensive study for deduced analogy between the two suggested systems. The presented approach takes into consideration two common biological features, behavioral responsive action as well as learning brain/ neural system function. More specifically, these systems are originated from Computational Biology approach, and associated to two non-neural and neural biological systems. These are: swarm intelligence performance of an Ant Colony System (ACS) [1], and observed psycho-learning experimental results of Pavlov's [2], and Thorndike's works [3]. That approach in our opinion seems to adopt implicit principles of biological information processing [4,5]. The two suggested systems given in the above are specifically presented through comparative study between distributed behavior function of ACS model [6,7], and Artificial Neural Network(ANN) modeling for Pavlov's learning process [8]. Previously, some investigational studies of analogy between of some natural and / or simulated systems were reported. Briefly, a sample set of such previous studies is reviewed as follows:

Analogy between simple invertebrate neural system and human brain function presented at [9]. Therein, the presented analogy concerned with learning and memory phenomena. Evolutional analogy between genetically reformed brainier mouse and human brain resulted in some promising recommended medical treatment for some cases of human learning disabilities [10,11,12]. Learning disabilities phenomena have been studied through its analogy with ANN simulating learning systems that along 
with suggestion for medical promising treatment of synaptic plasticity connections [13]. Furthermore, one piece of research that comparatively addressed the learning performance evaluation analogy between of both Pavlov's and Thorndike's experimental has been published at [14]. Additionally in educational field, the analogy between naturally observed phenomena due to noisy contaminated information. That is provided by teachers in classrooms to students versus ANN modeling while learning process is performed under contaminated noisy data has been well evaluated in details at $[15,16,17]$. Conclusively, it seems realistic to consider commonly both suggested systems as biological learning schematic models. These models adopted the principle of creatures' behavioral learning by interaction with environment [18].

In Figure 1 given in the below modeling of the adopted learning principle is illustrated after some published work [19]. Therein, the analogy of Hebbian coincidence learning rule $[20,21]$ with the optimum method for teaching how to read process is investigated [22].

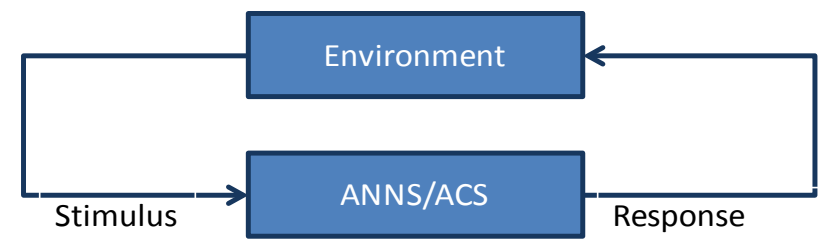

Figure 1. Shows how both systems modeling interact with environmental conditions considering that inputs stimuli and outputs response signals adapted from [19]

Considering some details for both systems on naturally biologically basis are presented as follows. Firstly, real ant colony system is capable of learning how to find the shorts path from a food source to their nest and vice versa. This functional learning process is performed well by adaptive interaction by environment. The food storage process is a surviving function adopts some trail updating rules for ant's pheromone following an algorithm reported at [6]. Furthermore, there are different versions of ant systems they proposed and presented in details at [23,24,25] that is namely ant density, ant quantity and ant cycle.

Interestingly, the other biological neural system is inspired by animal learning for how to respond correctly to some environmental input as external conditioned stimulus. That is observed by referring to results obtained after Pavlov's and Thorndike's experimental work [2,3]. Thus the animal seems to be responding obligatory for waiting expected food presentation. That resulting from the stored memorized experience during training phase updating its synaptic connectivity.

Herein, biologically distributed non-neural system considered by ACS is presented as a form of work in simulated world inhabited by mathematical modeling of artificial ants [25]. That is to try to solve TSP by cooperative communicative learning [1]. However, the biological neural system is studied in the basis of ANN modeling applied to simulate Pavlov's learning process $[8,14,27]$.

Commonly, both systems are motivated naturally by incentive desire to survive through adaptation with external live environmental conditions [2,3,6,7,27,28]. In other words, all of animals' systems are commonly behaved interactively with their external environment on the bases of biological hypothesis: "Creatures have a tendency to behave adaptively in order to keep survive." [18]. That behavior considered by either storage of food till facing hard expected life conditions at ACS model $[1,6]$, or by expecting picking food to save life at both Pavlov's and Thorndike's models [2,3,8,14]. The presented investigational analysis of comparative analogy between both systems results in two forms of adapted analogy directions namely the learning algorithms and output learning performance curves.

The rest of this paper is organized as follows. At the next second section, a brief review of first learning algorithm concerned with three neural behavioral learning systems' models is introduced. These are Pavlov's, Thorndike's experimental works' results. In addition to mouse's behavioral learning during its movement inside figure of eight (8) maze aiming to solve reconstruction problem. Performance of ACS behavioral learning model is briefly reviewed at third section, including cooperation and intercommunication among ants. At the fourth section, simulation results in addition to mathematical formulations are presented considering the comparison between neural and non-neural systems. Analogy between Neural and Nonneural Systems is given in two subsections. In Subsection 5.1, learning algorithms analogy however in subsection 5.2, learning convergence processes' shown to obey the least mean square error function. Finally, at the last sixth section, some conclusions and valuable discussions are introduced.

\section{Performance Analysis for Computational Intelligence (Neural Behavioural LearningSystems}

This section deals with analysis of three neural systems' performances. So, it is divided into basic three subsections. At subsections 2.1, 2.2 revising of Pavlov's and Thorndike's experimental works are introduced respectively. Moreover, at subsection 2.3 mouse's trials are presented during its movement inside figure of eight (8) maze aiming to solve reconstruction problem. Referring to Figure 2, it presents an overview for interactive learning model, and it is qualified to perform simulation of generalized learning model deduced from the above (Figure 1).

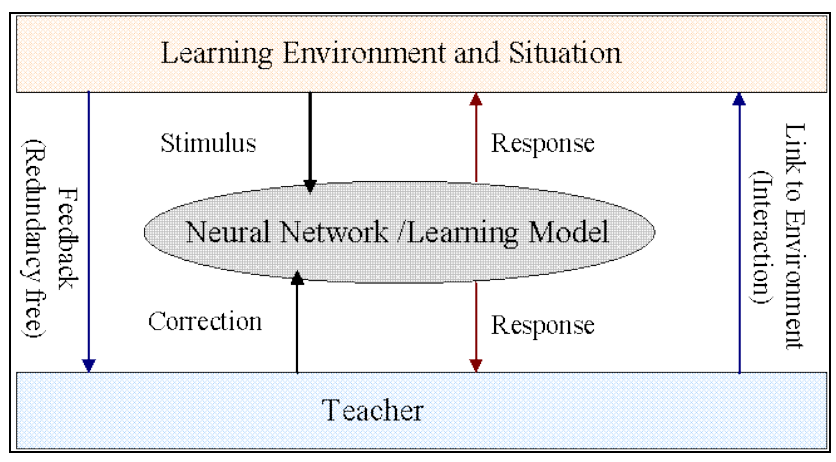

Figure 2. Illustrates generalized simple block diagram for interactive learning process. (Adapted from [17])

\subsection{Revising of Pavlov's work $[2,8,14]$}


The psycho-experimental work of Pavlov is known for classical conditioning. It is characterized by following two aspects: A spontaneous reaction that occurs automatically to a particular stimulus, and to alter the "natural" relationship between a stimulus and a reaction response was viewed as a major breakthrough in the study of behavior $[18,28,29]$. By referring to the original Pavlov's work, let us define what is meant by latency time. This time is briefly, defined as the delay period elapsed since acquisition of two input stimulating signals (pairings), till developing output response signals [2]. In more details, responding signals are held to be of zero value during their correlated latency time periods. Hence, by the end of these periods, output actions are spontaneously developed in a form of some number of salivation drops representing response signals intensities. These intensities observed to be in proportionality with the increase of the subsequent number of trials. So, this relation agrees with odd sigmoid function curve as reaching saturation state [8,14]. Conversely, on the basis of Pavlov's obtained experimental results, it is well observed mathematical interrelationship between latency time period versus subsequent number of trials can be illustrated explicitly in the form of hyperbolic function curve that mathematically expressed by equation:

$$
t(n)=\frac{\alpha}{n^{\beta}}
$$

where $\alpha$ and $\beta$ are arbitrary positive constant in the fulfillment of some curve fitting to a set of points as shown by graphical relation illustrated at Figure 3 in blow [30]. Noting, this figure illustrates some decay curve performance that represents latency time performance that curve has to be changed following individual differences of dogs. Similarly, by referring to Figure 4, the performance curve that represents relation between number of trial cycles versus the obtained output salivation drops' signals after receiving inputs stimuli by neural system. This relation performance curve has been obviously changes in accordance with individual differences of dogs.

\section{Latony time, sec}

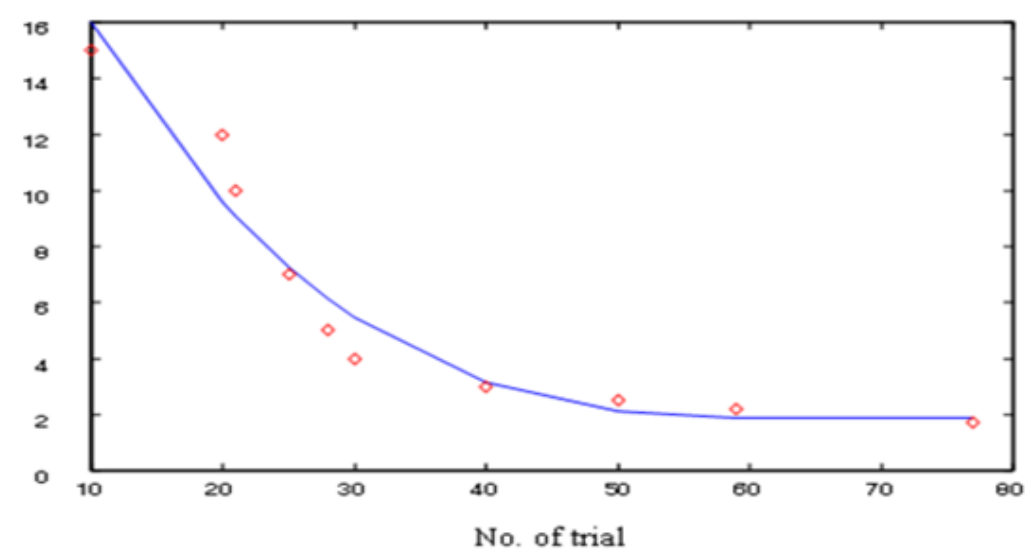

Figure 3. Observed latency time results after Pavlov's experimental work is presented by a fitting curve

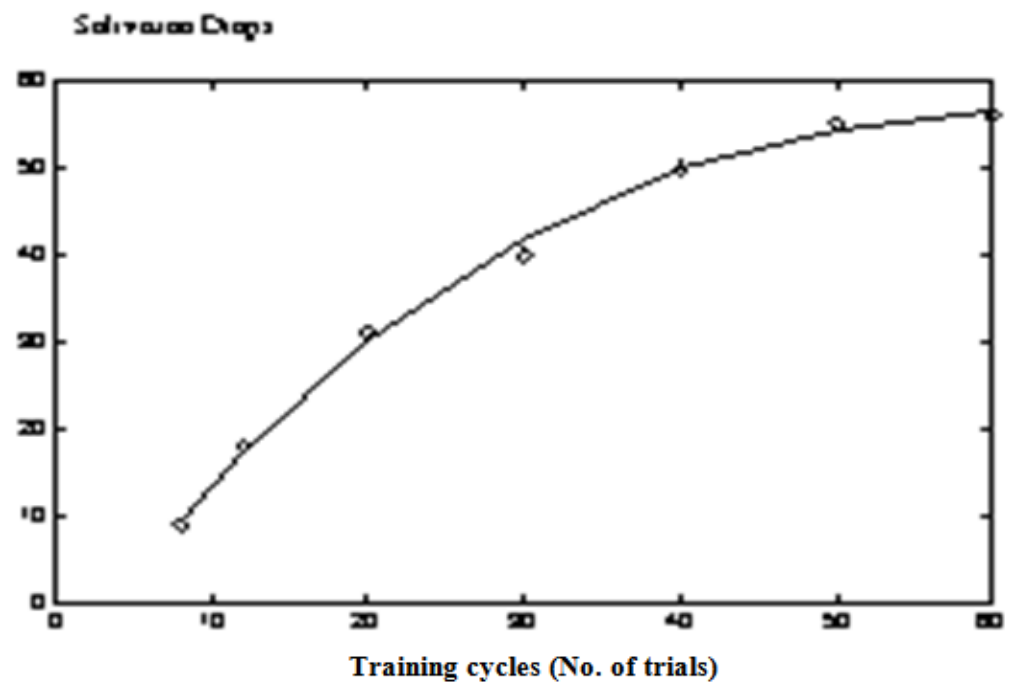

Figure 4. Illustrates the relation between number of trial cycles versus the obtained output salivation drops' signals after receiving inputs stimuliby neural system

\subsection{Revising of Thorndike's Work [3,14]}

Referring to behaviorism learning theory presented at [28], Thorndike had suggested three principles, which instructors (who adopted teaching based on behaviorism learning theory) should apply in order to promote effectiveness of behavioral learning process. These principles are given as follows. Furthermore, building on 
these he proposed an alternative teaching technique called programmed learning/instruction and also a teaching machine that could present programmed material. Initially, trials performed by cat results in observed random outputs' performance. By sequential trials, following observed errors become minimized, by the increased number of training (learning) cycles. Referring to Figure 4, original results of Thorndike's work is illustrated. This figure presents the relation between response time and number of trials. Furthermore, referring to that original Thorndike's experimental results given at Figure 4, represent behavioral learning performance of Thorndike's work. However, normalized learning curve that presents performance curve of experimental work is given approximately at Figure 5. Interestingly, the comparative analogy between performance curves of Pavlov's and Thorndike's work shown to behave similar to each other [14]. In general, principle of adaptive learning process (observed during creatures' interaction with environment) illustrated originally at [18].

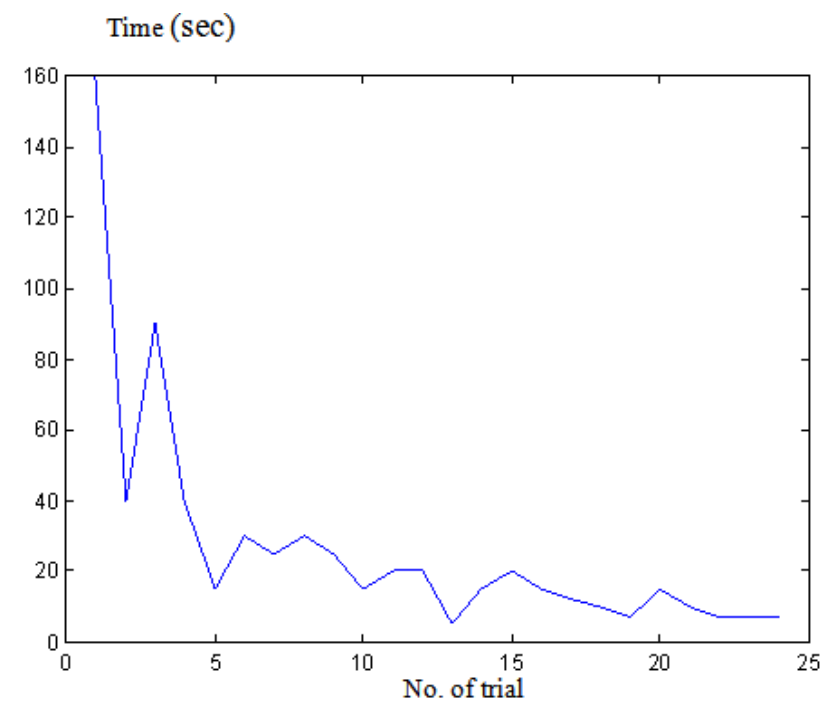

Figure 5. Original results present Thorndike's learning performance for a cat to get out from the cage for reaching food

Normalized Time
Response (Cycles)

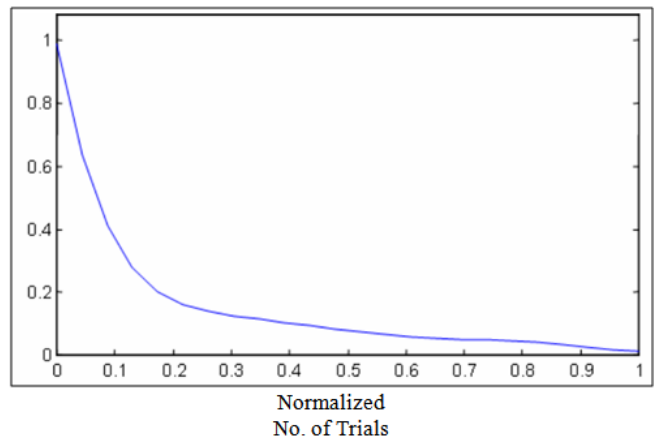

Figure 6. Thorndike's normalized results seem to be closely similar to exponential time decay

Referring to Figure 7, it observed that by increasing number of training cycles, the first learning algorithm converges to some fixed limiting values (for normalized time response). That observed results consider normalization of both number of trials values versus their corresponding normalized time response (for both original experimental work of Pavlov and Thorndike given at Figure 4 \& Figure 5 respectively).
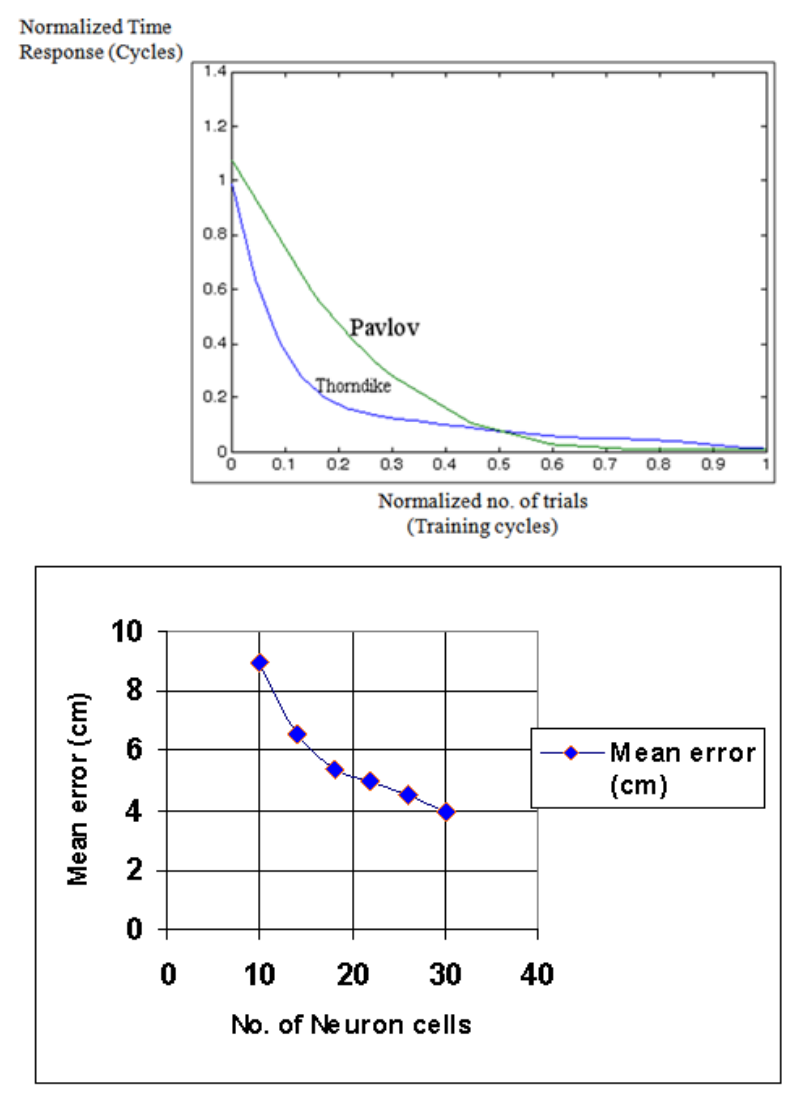

Figure 7. Comparison between Pavlov's and Thorndike's works considering normalization of obtained experimental results, the dashed line indicates the approach to Cramer-Rao bound based on Fisher information adapted from [32]

\subsection{Mouse's learning for Solving Reconstruction Problem}

Referring to [31], the timing of spikes in a population of neurons can be used to reconstruct a physical variable is the reconstruction of the location of a rat in its environment from the place fields of neurons in the hippocampus of the rat. In the experiment reported here, the firing part-terns of 25 cells were simultaneously recorded from a freely moving mouse [32]. The place cells were silent most of the time, and they fired maximally only when the animal's head was within restricted region in the environment called its place field [33]. The reconstruction problem was to determine the rat's position based on the spike firing times of the place cells. Bayesian reconstruction was used to estimate the position of the mouse in the Figure 8 maze shown at Figure 7, which adapted from [34]. Assume that a population of $\mathrm{N}$ neurons encodes several variables $\left(x_{1}, x_{2} \ldots \ldots\right)$, which will be written as vector $x$. From the number of spikes $\mathrm{n}=\left(n_{1}, n_{2}, \ldots \ldots n_{\mathrm{N}}\right)$ fired by the $N$ neurons within a time interval $\tau$, we want to estimate the value of $\mathrm{x}$ using the Bayes rule for conditional probability:

$$
P(\mathrm{x} \mid \mathrm{n})=P(\mathrm{n} \mid \mathrm{x}) P(\mathrm{x}) / P(\mathrm{n})
$$

Assuming independent Poisson spike statistics. The final formula reads

$$
P(x \mid n)=k P(x)\left(\prod_{i=1}^{N} f i(x)^{n i}\right) \exp \left(-\tau \sum_{i=1}^{N} f i(x)\right)
$$


Where $k$ is a normalization constant, $P(\mathrm{x})$ is the prior probability, and $f_{\mathrm{i}}(\mathrm{x})$ is the measured tuning function, i.e. the average firing rate of neuron $i$ for each variable value $\mathrm{x}$. The most probable value of $\mathrm{x}$ can thus be obtained by finding the $\mathrm{x}$ that maximizes $P(\mathrm{x} \mid \mathrm{n})$, namely,

$$
\hat{x}=\arg \max P(x \mid n)
$$

By sliding the time window forward, the entire time course of $\mathrm{x}$ can be reconstructed from the time varyingactivity of the neural population.

This appendix illustrates well Referring to results for solving reconstruction (pattern recognition) problem solved by a mouse in Figure 8 maze [32,33]. That measured results based on pulsed neuron spikes at hippocampus of the mouse brain. According to following table, the error value seems to decrease similar to exponential curve decays to some limit value versus (place field) cells.

Table 1. Relation between number of cells and mean error in solving reconstruction problem

\begin{tabular}{|c|c|c|c|c|c|c|}
\hline No. of neuron cells & 10 & 14 & 18 & 22 & 26 & 30 \\
\hline Mean error $(\mathrm{cm})$ & 9 & 6.6 & 5.4 & 5 & 4.5 & 4 \\
\hline
\end{tabular}

Noting that, the value of mean error converges (by increase of number of cells) to some limit, excluded as Cramer-Rao bound. That limiting bound is based on Fisher's information given as tabulated results in the above and derived from [33]. That implies LMS algorithm is valid and obeys the curve shown at in blow.

Furthermore, it is noticed that the algorithmic performance learning curve referred to Figure 6, converged to bounding limit (of minimum error value) fixed Cramer Rao bound (Limiting value). That is analogous to minimum time response corresponding to maximum number of trials limit by referring to above Figure 7. Interestingly, considering comparison between learning curve performances at Figure 7 and learning at ACS (see Figure 11). It observed the analogy when comparing number of place field cells (at hippocampus mouse's brain area) versus the number of cooperative ants while searching for optimized TSP solution adopting ACS. More details are given herein at the fifth section where simulation results have been presented.

\section{Performance Analysis for Computational Intelligence (Non-neural Behavioural Learning System)}

This section deals with performance analysis of a nonneural system namely Ant Colony System, in three subsections as follows.

\subsection{Revising Ant Colony System Performance}

Herein, ACS considered as a non-neural biological system given at Figure 8 in below. Referring to this figure, ants' movements are going on a straight line that connects a food source to their nest (Figure 8A). It is well known that the primary means for ants to form and maintain the line is a pheromone trail. Ants deposit a certain amount of pheromone while walking, and each ant probabilistically prefers to follow a direction rich in pheromone. This elementary behaviour of real ants can be used to explain how they can find the shortest path that reconnects a broken line after the sudden appearance of an unexpected obstacle has interrupted the initial path (Figure 8B). In fact, once the obstacle has appeared, those ants which are just in front of the obstacle cannot continue to follow the pheromone trail and therefore they have to choose between turning right or left. In this situation we can expect half the ants to choose to turn right and the other half to turn left. A very similar situation can be found on the other side of the obstacle (Figure 8C). It is interesting to note that those ants which choose, by chance, the shorter path around the obstacle will more rapidly reconstitute the interrupted pheromone trail compared to those which choose the longer path. Thus, the shorter path will receive a greater amount of pheromone per time unit and in turn a larger number of ants will choose the shorter path. Due to this positive feedback (autocatalytic) process, all the ants will rapidly choose the shorter path (Figure 8D). The most interesting aspect of this autocatalytic process is that finding the shortest path around the obstacle seems to be an emergent property of the interaction between the obstacle shape and ants distributed behaviour: Although all ants move at approximately the same speed and deposit a pheromone trail at approximately the same rate, it is a fact that it takes longer to contour obstacles on their longer side than on their shorter side which makes the pheromone trail accumulate quicker on the shorter side. It is the ants' preference for higher pheromone trail levels which makes this accumulation still quicker on the shorter path. This process is adapted with the existence of an obstacle through the pathway from nest to source and vice versa, however, more detailed illustrations are given through other published research work, [1].Therein, ACS performance obeys computational biology algorithm used for solving optimally travelling salesman problem TSP [1].

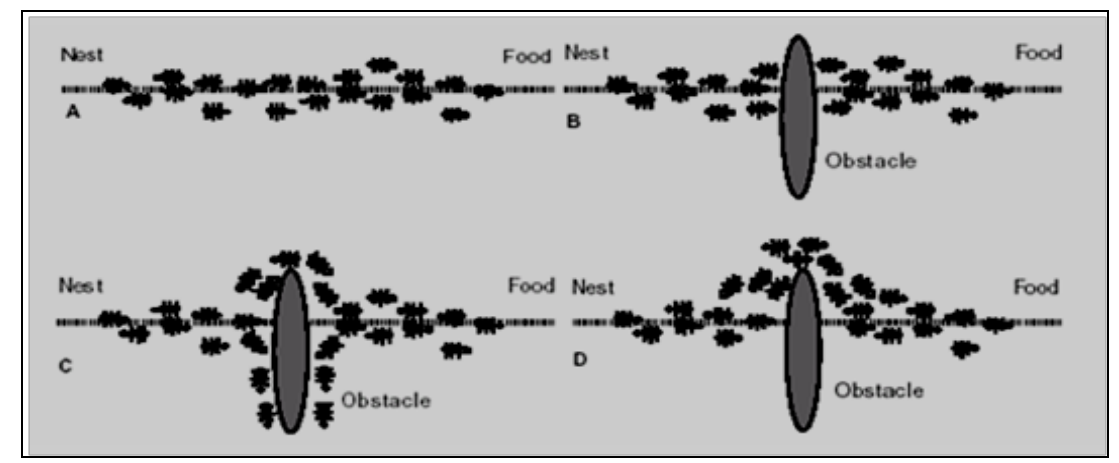

Figure 8. Illustrates the process of transportation of food (i.e., from food source) to food store i.e., nest (A). This process is adapted with the existence of an obstacle through the pathway from nest to source and vice versa (B). Detailsabout rapidly choice of the shorter path have been given at [1] 
Referring to two more recent research [35,36], an interesting view distributed biological system ACS is presented. Therein, the ant Temnothoraxalbipennis uses a learning paradigm (technique) known as tandem running to lead another ant from the nest to food with signals between the two ants controlling both the speed and course of the run. That learning paradigm involves bidirectional feedback between teacher and pupil and considered as supervised learning [36,37,38].

ACS optimization process has been presented versus mice's reconstruction problem. The relation between cooperative process in ACS as illustrated at Figure 2 in the above and activity at hippocampus of the mouse brain is illustrated in details at two recently published works $[39,40]$.

\subsection{Cooperative Learning by ACS for Solving TSP}

Cooperative learning by Ant Colony System for solving TSP Referring to Figure 10, which adapted from [1], the difference between communication levels among agents (ants) develops different outputs average speed to optimum solution. The changes of communication level are analogues to different values of $\lambda$ in sigmoid function as shown at equation (4) in below. This analogy seems to be well by referring to original Pavlov's experimental results [2] where the output salivation signal is increased depending upon the value of no of training cycles. When the numbers of training cycles increase virtually to an infinite value, the number of salivation drops obviously reaches a saturation value. Additionally the pairing stimulus develops the learning process turned in accordance with Hebbian learning rule [20,21]. However in case of different communication level values corresponding to gain factor $(\lambda)$ (at sigmoid function) other than zero implicitly means that output signal is developed by neuron motors.

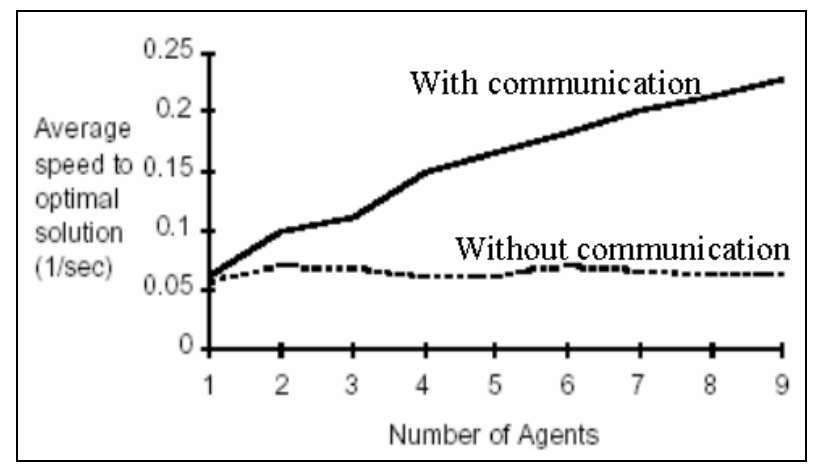

Figure 9. Illustrates performance of ACS with and without communication between ants \{adapted from [1]

In Figure 10, the difference between the communication levels among agents (i.e., ants) develops average speed on different outputs to optimum solution. This figure is adapted from [1] and modified with additional virtual curves representing different levels of communication among ants. Virtual extension leads to better average speed to reach optimum solution as illustrated to that figure.

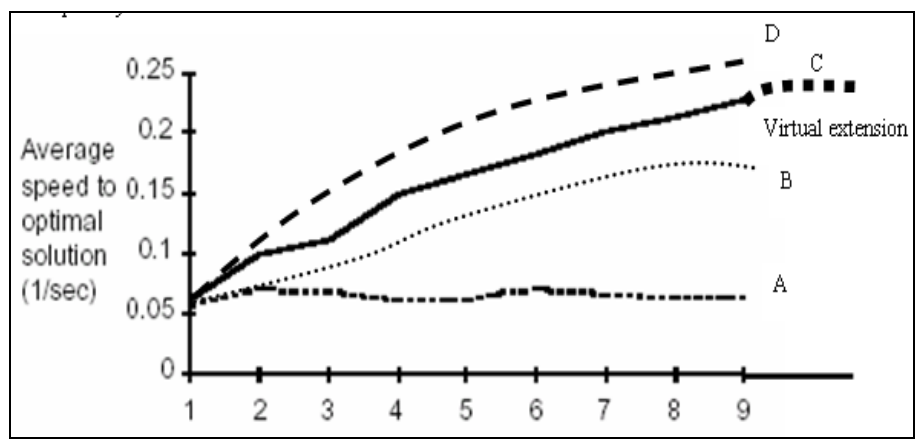

Figure 10. Communication determines a synergistic effect. Communication among agents. Different communication levels lead to different values of average speed as follows by curves $\mathrm{A}, \mathrm{B}, \mathrm{C}$, and D

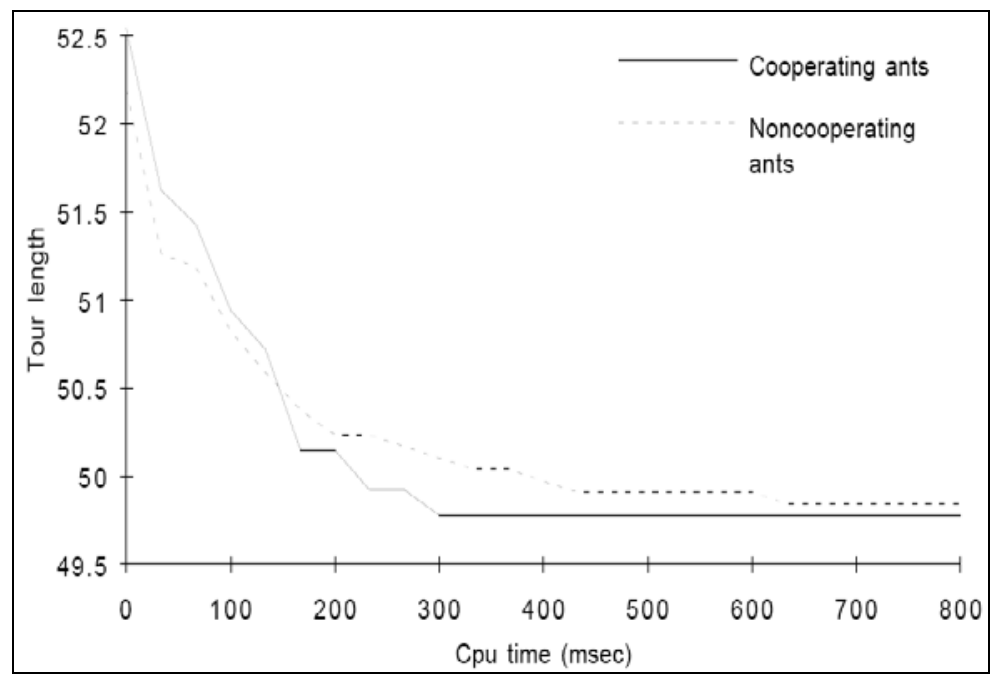

Figure 11. Cooperating ants find better solutions in a shorter time. Average on 25 runs. The number of ants was set to m=4, adapted from [24] 


\subsection{Intercommunication among Ants}

Referring to Figure 10 shown in below, the relation between tour lengths versus the CPU time is given. It is observed the effect of ant cooperation level on reaching optimum (minimum tour). Obviously, as level of cooperation among ants increases (better communication among ants) the CPU time needed to reach optimum solution is decreased. So, that optimum solution is observed to be reached (with cooperation) after 300 (msec) CPU the while that solution is reached after 600 (msec) CPU time (without cooperation).

Additionally, the Figure 12, it is essential to note that CPU time increasing means that stored experience increases that is analogous to number of trials increase by time during Pavlov's learning process as given at Figure 3.

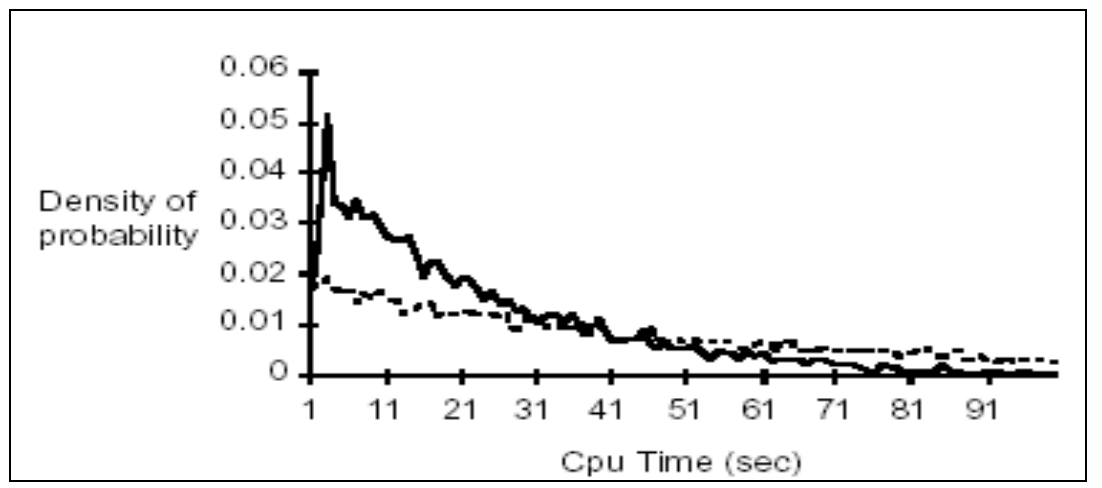

Figure 12. Intercommunication changes the probability distribution of first finishing times. Continuous curve represents with intercommunication, however dashed curve represents without intercommunication among ants

\section{Simulation Results and Mathematical Formulations}

This section deals with obtained simulation results after realistic modeling of neural system performance at subsection 4.1. In subsection 4.2 mathematical formulations is introduced for curves of both neural and non-neural systems' performances.

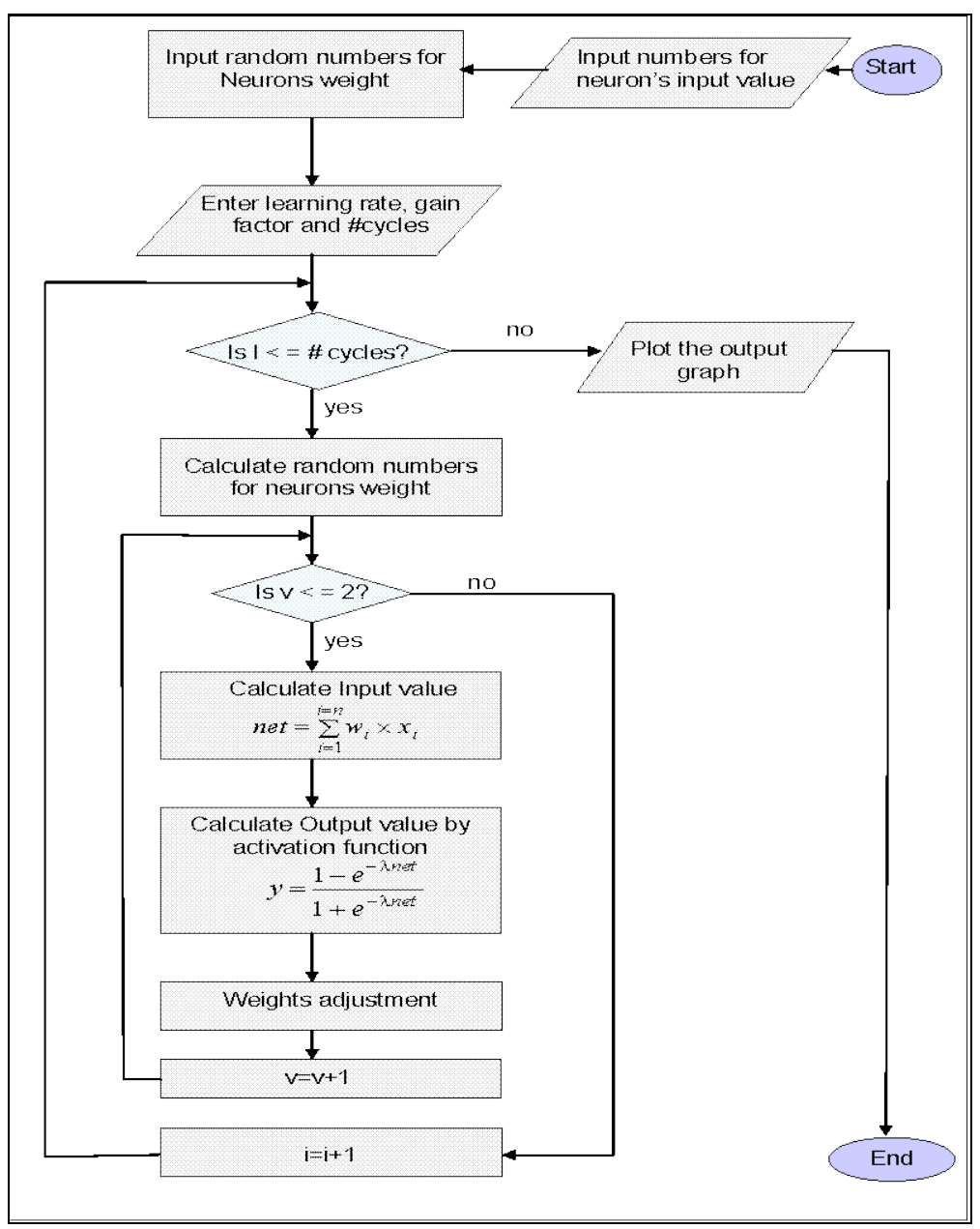

Figure 13. A simplified macro level flowchart describing algorithmic steps for Artificial Neural Networks modeling considering various neurons' number 


\subsection{Simulation Results}

Figure 13 introduces the flowchart for simulation program which applied for performance evaluation of behavioral learning processes. Considering the two adopted cases of biological creatures having either neural or non-neural systems. That Figure presents a simplified macro-level flowchart which describes briefly algorithmic steps for realistic simulation program of adopted Artificial Neural Networks' model for different number of neurons using. The results are shown at the two Figure 14, and Figure 15 after that program running. Referring to Figure 14, which has been adapted from [1], the difference between communication levels among agents (ants) develops different outputs average speed to optimum solution. The changes of communication level are analogues to different values of gain factor $(\lambda)$ in sigmoid function. This analogy seems very similar that performance given at Figure 4, where the output salivation signal is increased depending upon the value of no of training cycles. Assuming that the number of training cycles increases virtually to an infinite value, the number of salivation drops obviously reach a saturation value additionally the pairing stimulus develops the learning process turned in accordance with Hebbian learning rule [21]. However in case of different values of $\lambda$ other than zero implicitly means that output signal is developed by neuron motors.

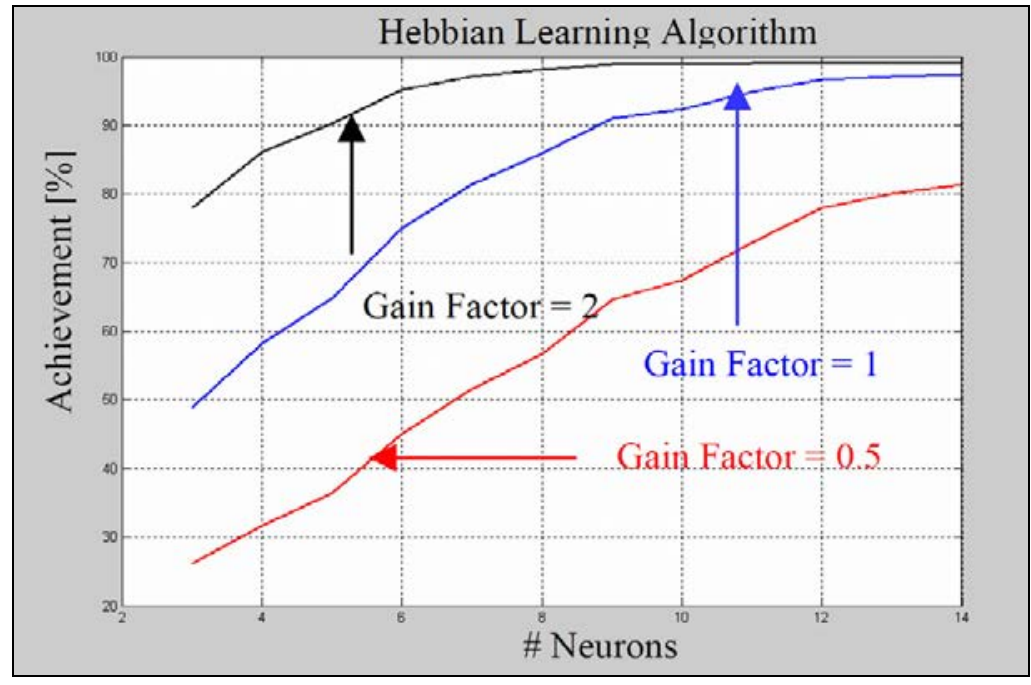

Figure 14. Illustrate learning performance to reach optimum solution considering different intercommunication levels corresponding to gain factors $0.05,1$, and 2

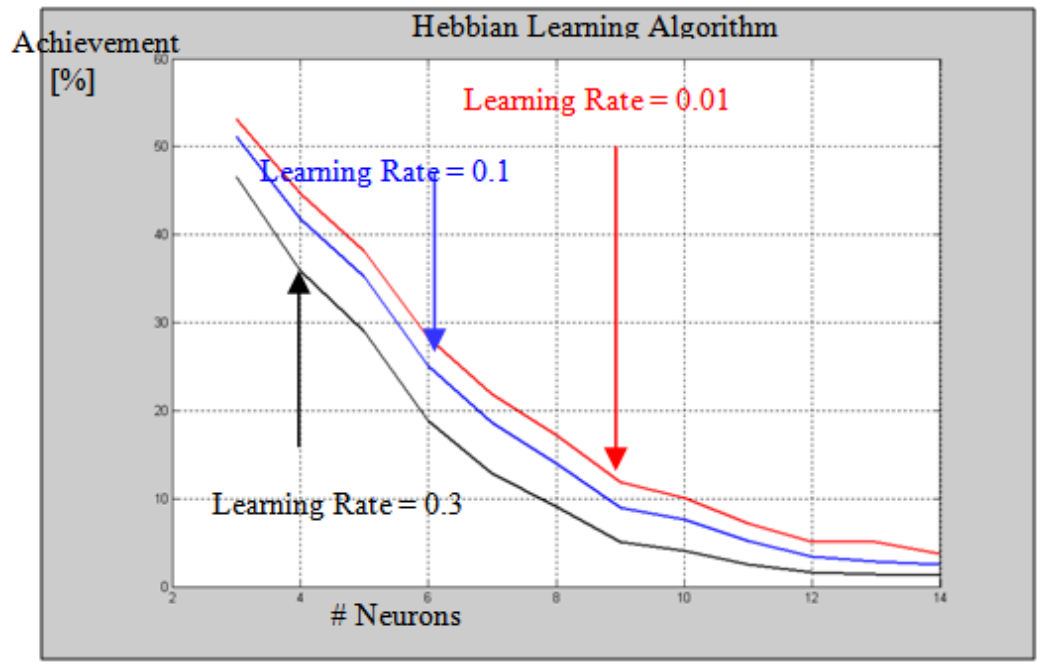

Figure 15. Illustrate learning performance error-rate with different learning rates $(0.01,0.1$, and 0.3$)$ which correspond to various noisy environmental conditions

\subsection{Mathematical Formulations}

This section aims to formulate mathematically effective contributions of two specific ANN design parameters. So, it considers deferent values of gain factors, and learning rates presented by Greek letters $(\lambda, \eta)$ respectively. Moreover, graphical presentations for suggested mathematical formulation contributed with different values of both parameters are shown at Figure 13, and
Figure 14 given in below. Additionally, the effect of both design parameters is observed either implicitly or explicitly on dynamical synaptic plasticity illustrated at weigh dynamics equations [20,33]. Additionally, normalized behavior model considers the changes of communication levels (indicated by $\lambda$ parameter). This parameter value causes changing of the speeds for reaching optimum solutions for Travelling Salesman Problem (TSP) using Ant colony System (ACS) 
$[1,23,24,25]$. The following equation presents a set of curves changes in accordance with different gain factor values $(\lambda)$.

$$
\mathrm{y}(\mathrm{n})=(1-\exp (-\mathrm{li}(\mathrm{n}-1))) /(1+\exp (-\mathrm{li}(\mathrm{n}-1)))
$$

Where $\lambda \mathrm{i}$ represents one of gain factors (slopes) of sigmoid function.
These curves represent a set of sigmoid functions to reach by time maximum achievement. Conversely, following formula where suggested $\left(\eta_{i}\right)$. It presents a set of normalized decay (negative exponential curves) for different learning rate values given by as follows:

$$
\mathrm{y}(\mathrm{n})=\exp \left(-\mathrm{h}_{\mathrm{i}}(\mathrm{n}-1)\right)
$$

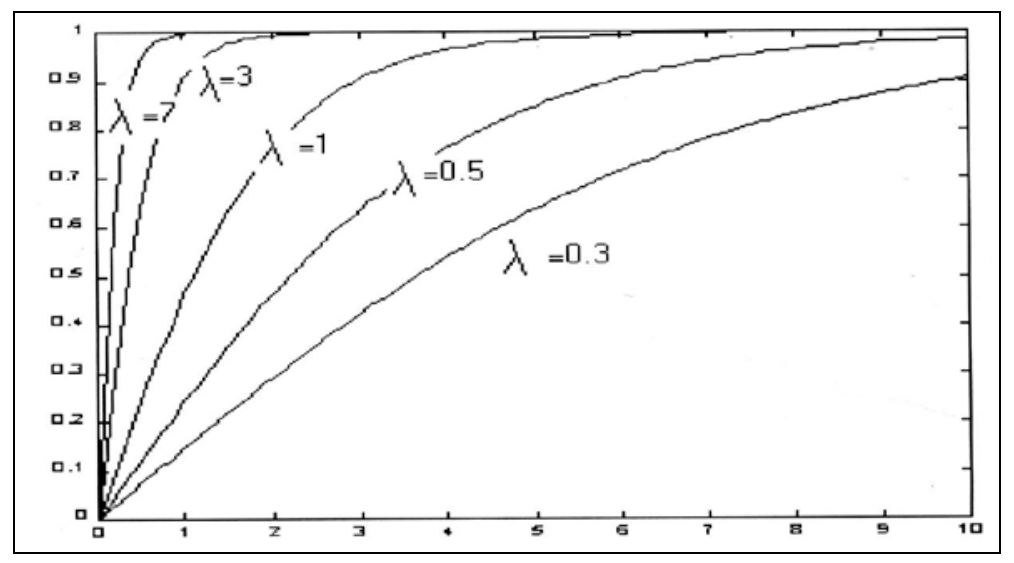

Figure 16. Graphical representation of learning performance of model with different gain factor values $(\lambda)$

Where (n) is the number of training cycles. That set of curves is illustrated graphically at Figure 8 given in blow. the examples given considering normalization of output response values.

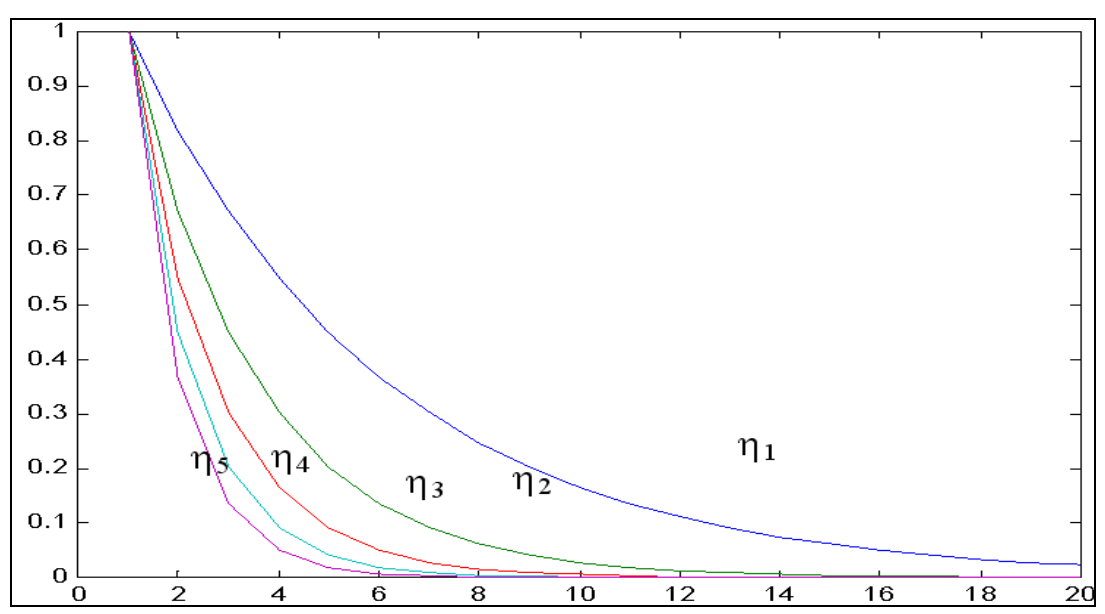

Figure 17. Illustrates different learning performance curves for different learning rate values

\begin{tabular}{|l|}
\hline Initialize \\
\hline Loop /* at this level each loop is called an iteration */ \\
Each ant is positioned on a starting node \\
Loop /* at this level each loop is called a step */ \\
Each ant applies a state transition rule to incrementally build \\
a solution and a local pheromone updating ruleUntil all ants have \\
built a complete solution \\
A global pheromone updating rule is applied // Until \\
End condition
\end{tabular}

Figure 18. illustrates ant colony algorithm in two loops with iterative learning cycles

\section{Analogy between Neural and Non-neural Systems}

\subsection{Learning Algorithms Analogy}

Referring to Figure 18, it presents the algorithmic steps for solving basically the Travelling Sales Man (TSP) considering the process of transportation of food (from food source) to food store (nest) shown at Figure 8. Interestingly, it clear that both algorithmic steps presented at Figure 18 and Figure 19 are analogous to each other. Furthermore, the algorithmic steps shown at Figure 9 are describing behavioural learning in Pavlov's iterative work processes based on neural network model presenting Hebbian learning as introduced at [8]. The results obtained 
after performing the original psych-experimental work concerned with Pavlov's dog, are nearly well analogous to the behavioural learning of mouse's trail for solving reconstruction problem That is illustrated during the while detailed comparative evaluation for learning creativity of Cats, Dogs, Ants, and Rats presented at [31].

\begin{tabular}{|l|}
\hline Initialize \\
\hline Loop /* at this level each loop is called an iteration that \\
completed by the end of learning process*/ \\
Each pairing stimulus is positioned on a starting latony time \\
cycle \\
Loop /* at this level each loop is called a step which completed by \\
developing some output by the motor neuron */ \\
Each weight is changed dynamically according to Hebbian \\
learning law \\
Until developing output signal corresponding to any arbitrary \\
latony time \\
A maximum salivation signal is obtained when threshold value \\
reaches to zero // Until \\
End condition
\end{tabular}

Figure 19. illustrates training process in ANN models considering latency time phenomenon having two loops with iterative learning cycles

\subsection{Least Mean Square LMS Algorithm [20]}

At the Figure 20, it presents the learning convergence process for least mean square error as used for training of ANN models [20]. It is clear that this process performed similarly as ACS searching for minimum tour when solving TSP [1]. Furthermore, it obeys the learning performance observed during psycho experimental work carried for animal learning [2,3,29].

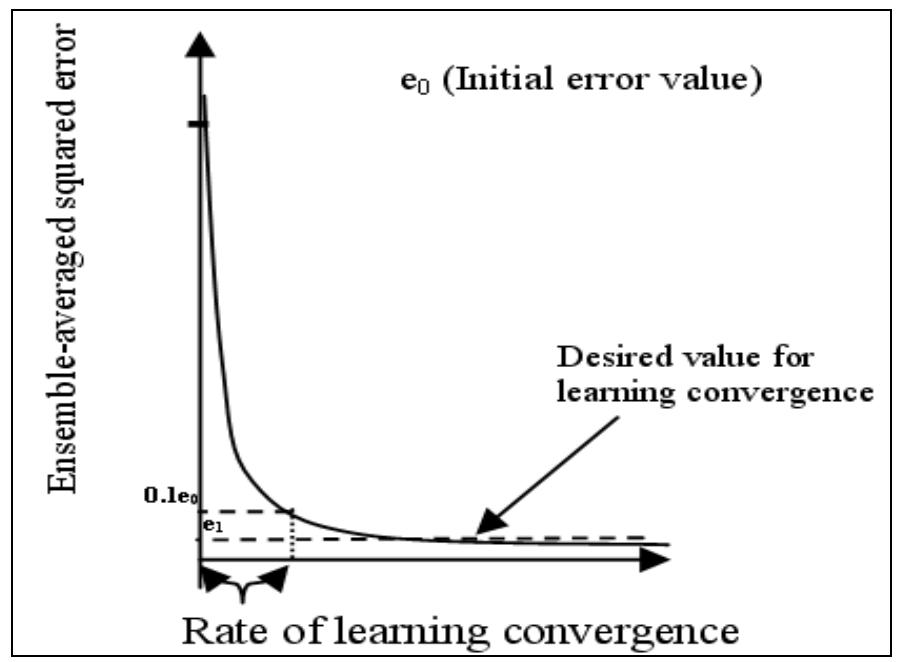

Figure 20. Idealized learning curve of the LMS algorithm adapted from [20]

\section{Conclusions and Discussion}

According to above animal learning experiments (dogs, cats, and mice), and their analysis and evaluation by $\mathrm{ANN}^{\mathrm{S}}$-modeling, all of them seen to be in well agreement with that optimization process by ACS. Also, the performance of both (ant and animals) is similar to that for latency time minimized by increasing of number of trials. Referring to Figure 7, it is shown that both learning performance curves presenting both work for Thorndike and Pavlov are commonly characterized by their hyperbolic decay and also, both obeys generalized (LMS) for error minimization by learning convergence. In this context, that algorithm agrees with the behavior of brainier mouse behavior (that is genetically reformed as illustrated at [11]. Generally, the four introduced nonhuman (neural and non-neural) models in this work perform their behavioral learning functions similar to LMS error algorithm, which introduced at subsection 5.2. By some details, artificial neural network models either performing computation on analogue signaling base or on pulsed spikes decoding criterion, they both leads to learning convergence following LMS error algorithm. Noting that, reconstruction method following Bayesian rule is bounded to Cramer Rao's limit. This limit is analogous to minimum response time in Pavlov experiment, and Thorndike work as well. Similarly, for ACS, optimization processes are following as LMS error algorithm when performing solution TSP. Additionally; adaptation equations for all of three systems are running in agreement with dynamic behavior of each other. Additionally, the learning algorithms for the presented four models are close to each other with similar iterative steps (either explicitly or implicitly). Finally, it is worthy to note that the rate of increase of salivation drops is analogous to rate for reaching optimum average speed in 
ACS optimization process. Similarly, this rate is also analogous to speed of cat getting out from cage in Thorndike's experiment. Noting that, increase on number of artificial ants is analogous to number of trials in Pavlov's work. Some studies related to the analogy between some biological system invertebrate and mammalians leads to interestingly practical and theoretical future research trends. For example, the analogy between Hebbian coincidence learning rule and teaching how to read [19]. Moreover, the study of analogy between human brain functions and genetically engineered brainier mouse supports new trends for medical treatment of some human learning disabilities [13].

\section{References}

[1] Dorigo, M., \& Gambardella, L. M. (1997). Ant colony system: a cooperative learning approach to the travelling salesman problem. IEEE Transactions on Evolutionary Computation, 1(1), 53-66. available online at: www.iridia.ulb.ac.be/dorigo/aco.html.

[2] Pavlov, I.P.: Conditional Reflex, An Investigation of The Psychological Activity of the Cerebral Cortex. New York, Oxford University press, (1927).

[3] Thorndike, E.L.: Animal Intelligence, Darien, Ct. Hafner, (1911).

[4] Rumelhart, D.E., Mccland, J.I.: eds. Parallel distributed processing: exploration in microstructure of cognition. vol 1 Cambridge, M.A: MIT press (1986).

[5] Lippman, R.: An introduction to computing with neural nets. In: IEEE-ASSP magazine April pp. 4-20(1987).

[6] Bonabeau, E., Theraulaz, G,.: Swarm smarts, MajallatAloloom. vol 17, No 5, pp 4-12,and references therein May (2001).

[7] Garnier, S., Gautrais, J., Theraulaz, G.: The biological principles of Swarm Intelligence. In: Swarm Intelligence Journal Volume 1, Number 1 / pp.3-31, June, (2007).

[8] Hassan H. and Watany, M.: On Mathematical Analysis of Pavlovian Conditioning Learning Process using Artificial Neural Network Model. In: 10th Mediterranean Electrotechnical Conf., May 29-3, Cyprus(2000).

[9] Kandel E., Small systems of neurons. In: Scientific American, vol 3, (1979).

[10] Bliss, T.V.P., Lomo, T.: Lpng lasting potentiation of synaptic transmission in the dentate area of anaesthetized rabbit following stimulation of perforatant path. In: Journal of Physiology, vol 232 (1973).

[11] Tsien, J, Z.: Building Of a Brainer Mouse, MajallatAloloom, vol 17, May (2001).

[12] Javitt, D. C., Coyle, J.T.: Decoding Schizophrenia. In: MajallatAloloom, vol 20, (2004).

[13] Ghonimy M. A., et al.: Learning ability in neural network model. In: Second International Conference On Artificial Intelligence Application, Cairo, Egypt, 400-413, Jan. 22-24, (1994).

[14] Hassan H., Watany, M.: On comparative evaluation and analogy for pavlovian and throndikian psycho-learning experimental processes using bioinformatics modeling, In AUEJ (2003).

[15] Hassan, M., H.: Application of Neural Network model for Analysis and Evaluation of Students Individual Differences. In: Proceeding of ICEENG Conference (1998).

[16] Ghonaimy M.A., Al - Bassiouni, A.M. and Hassan, H.M "Leaning Of Neural Networks Using Noisy Data”. Second International Conference on Artificial Intelligence Applications, Cairo, Egypt, Jan 22-24, 1994. PP. 387-399.

[17] Hassan, H. M "On Analysis and Evaluation of Non-Properly Prepared Teachers Based on Character Optical Recognition Considering Neural Networks Modeling" Published at Proceedingsof the International Conference on Pattern Recognition and Image Processing ((ICPRIP'15) that will take place on March 16-17, 2015 Abu Dhabi (UAE).

[18] Fukaya, M., et.al.: Two level Neural Networks: Learning by Interaction with Environment. In: 1st ICNN, San Diego (1987).

[19] Hassan, H. M.: On mathematical analysis, and evaluation of phonics method for teaching of reading using artificial neural network models. In: SimMod simulation and modeling conference (2005).
[20] Haykin S.: Neural Networks, Englewood Cliffs, NJ:PrenticeHallpp 50-60, (1999).

[21] D.O. Hebb, “The organization of behaviour”, Wiley, New York (1949).

[22] Rayner, K., Foorman, B., R., Perfetti, C, A., Pesetsky, D., Seiedenberg, M, S.: How Should Reading Be Taught? In: MajallatAloloom, vol 19, number 6/7 4-11(2003).

[23] Dorigo, M.: Optimization learning and natural algorithms, (in Italian), Ph.D Thesis Dip. Electronico, Politecnico di Milano, (1992).

[24] Eric Bonabeau, Marco Dorigo and Guy Theraulaz, Swarm Intelligence: fromnatural to artificialsystems, Oxford UniversityPress, 1999.

[25] Dorigo, C, M., Maniezzo, V.: Distributed optimization by Ant Colonies. In: Proceedings of The First European conference on Artificial Life (ECAL 91), pp 134-142 Elevier, (1991).

[26] H. M. Hassan. "On principles of biological information processing concerned with learning convergence mechanism in neural and non-neural bio-systems", Published at CIMCA, 28-30 Nov. 2005.

[27] Grossberg, S., Levine, D., S.: Neural dynamics of attentionallymodulated Pavolvian conditioning: Blocking, inter-stimulus, interval and secondary reinforcement. In: Applied Optics (1987).

[28] Gayla S. Keesee "Learning Theories" Available online at http://teachinglearningresources.pbworks.com/w/page/19919565/ Learning\%20Theories.

[29] Hampson, S.E. Connectionistic Problem Solving, Computational Aspects of Biological Learning, Berlin, Birkhouser, 1990.

[30] H.M.Hassan, Ayoub Al-Hamadi, " On Comparative Analogy Between Ant Colony Systems and Neural Networks Considering Behavioral Learning Performance" published at the 4th Indian International Conference on Artificial Intelligence (IICAI-09) held in Tumkur (near Bangalore), India on December 16-18, 2009.

[31] Hassan, M.H., 2008 " A Comparative Analogy of Quantified Learning Creativity in Humans Versus Behavioral Learning Performance in Animals: Cats, Dogs, Ants, and Rats.(A Conceptual Overview), published at WSSEC08 conference held on 18-22 August 2008, Derry, Northern Ireland.

[32] Zhang et al, 1998: Interpreting neuronal population activity by reconstruction.” Journal of Neurophysiology, 79:1017-44.

[33] Wilson, M. A. and McNaughton, B. L. Dynamics of the hippocampal ensemble code for space. Science, 261: 1055-8.

[34] Hassan M. H. Mustafa,et.al. "Comparative Analogy of Neural Network Modeling Versus Ant Colony System (Algorithmic and Mathematical Approach)" Published at the proceeding of International Conference on Digital Information Processing, EBusiness and Cloud Computing (DIPECC 2013) that held in Dubai, UAE on October 23-25 2013. Available online at: http://sdiwc.net/conferences/2013/dipecc2013/.

[35] H.M. Hassan, "On Mathematical Modeling of Cooperative ELearning Performance During Face to Face Tutoring Sessions (Ant Colony System Approach)"published at IEEE EDUCON 2011, on Education Engineering-Learning Environments and Ecosystems in Engineering Education, held on April 4-6, 2011, Amman, Jordan. Available on line at: http://www.google.com.sa/url?sa $=t \& r c t=j \& q=\& e s r c=s \& f r m=1 \& s$ ource=web\&cd=9\&ved=0CHQQFjAI\&url=http\%3A\%2F\%2Fedit lib.org\%2Fd\%2F45687\&ei=rsUFU66SAcc0wWakIHAAQ\&usg=AFQjCNFXdog2WcQE_3DE58sVp7aaVH4Lw.

[36] Rechardson T.,Franks N.R. "Teaching in tandem-running ants", Nature, Jan 2006,12;439(7073):153.

[37] Eric Bonabeau, and Guy Theraulaz, "SwarmSmarts" MajallatAloloom, May 2001, vol 17, No 5, pp 4-12.

[38] Eric Bonabeau, Marco Dorigo and Guy Theraulaz, Swarm Intelligence: fromnatural to artificialsystems, Oxford UniversityPress, 1999.

[39] H.M. Hassan, "Comparative Performance Analysis for Selected Behavioral Learning Systems versus Ant Colony System Performance (Neural Network Approach)". Published at the International Conference on Machine Intelligence ICMI 2015. Held on Jan 26-27, 2015, in Jeddah, Saudi Arabia.

[40] H.M. Hassan, et.al. "Comparative Performance Analysis and Evaluation for One Selected Behavioral Learning System versus an Ant Colony Optimization System" Published at the Proceedings of the Second International Conference on Electrical, Electronics, Computer Engineering and their Applications (EECEA2015), Manila, Philippines, on Feb. 12-14, 2015. 